\title{
Absence of Biochemical Activity
}

National Cancer Institute

\section{Source}

National Cancer Institute. Absence of Biochemical Activity. NCI Thesaurus. Code C45422.

Indicates an absence of a measurable amount of a specific function or role performed by a biological entity in a biophysical process, biochemical reaction, or biochemical process. 